\title{
Diagnostic Accuracy of mammography Versus Distribution of p63 in Fine Needle Aspiration of Breast Malignancies
}

\author{
${ }^{1}$ Dr Neha Tyagi, ${ }^{2}$ Dr Charanjeet Ahluwalia, ${ }^{3}$ Dr Sachin Kolte, ${ }^{4}$ Dr Akansha, \\ ${ }^{5}$ Dr Neha Antil, Dr Himani Bhankar ${ }^{6}$ \\ ${ }^{1,4,6}$ Senior Resident, Department Of Pathology \\ ${ }^{5}$ Senior Resident,Department Of Radiology \\ ${ }^{2,3}$ Associate Professor, Department Of Pathology
}

\begin{abstract}
Mammography is used as a screening tool for detection of carcinoma breast above the age of 35 years. FNAC is an established technique for the detection of breast malignancies. Myoepithelial cells are important marker for identification of breast carcinoma. p63 immunostaining has gained wide popularity for identification of myoepithelial cells. We planned this study to compare the effectiveness of mammography and p63 in detection of carcinoma breast.Total 50 cases were included in the study. Clinical features, mammography, FNAC and p63 immunostaining were performed. Results of mammography and p63 immunostaining were compared with the FNAC diagnosis. These were analysed statistically using chi-square test.Infiltrating ductal carcinoma (IDC) was found in 54\% of cases. 96\% IDC cases were p63 negative when stained immunocytologically. Diagnostic accuracy of p63 was significantly higher than mammography for diagnosing breast malignancies ( $p$ value <0.05).p63 can be a good adjunct to FNAC in diagnosis of breast malignancies. However, the cut off value of percentage of p63 positive cells needs to be standardised.
\end{abstract}

Keywords: immunostaining, infiltrating ductal carcinoma ,mammography, myoepithelial, p63.

\section{Introduction}

FNAC is a simple,inexpensive,rapid and widely acceptable tool for accurate diagnosis of benign as well as malignant lesions of breast (1). It has both high sensitivity as well as specificity with one of the study showing sensitivity of $84.4 \%$ and specificity of $99.5 \%$ in about 2000 cases (2). However, FNAC being a cytological diagnosis there are chances of false negative and false positive results (3). Breast lesions which are very small and difficult to palpate can be picked up by mammography (4). However, sensitivity of mammography alone is $98 \%$ in fat replaced breast and about $50 \%$ in dense breast thereby giving variable results (5).

Inspite of the growing popularity of core needle biopsy, FNAC still holds a role in "Triple assessment" of the patients (1). Triple test technique has shown $100 \%$ diagnostic accuracy in some studies (2). There arises situation when it becomes very difficult to provide a definitive diagnosis in breast lesions with the help of FNAC. The lesions which pose dilemma in diagnosis includes proliferative breast disease, papillary breast tumours and few well-differentiated carcinoma including tubular carcinoma (1). They can be referred to as "Grey Zone" lesions. Cytologically it is very difficult to differentiate ductal carcinoma in situ (DCIS) from invasive carcinoma (1).

The presence of myoepithelial cells is considered a key feature to distinguish benign from malignant lesions. They are retained in most of the benign breast lesions and are lost in malignancies. At times, it becomes difficult to differentiate them from apoptotic cells, epithelioid histiocytes and stromal cells in cytology (1).

Various immunocytochemical markers available to demonstrate myoepithelial cells includes calponin, smooth muscle myosin heavy chain, hcaldesmon, CK5/6 (6). These markers are useful in histological specimens but unreliable in cytological specimen as they stain fibroblasts and vascular walls as well (3).p63, a nuclear marker is a specific and reliable marker for myoepithelial cells in breast FNAC samples (7). The present study was undertaken to evaluate the potential diagnostic role of p63 immunostaining in comparison to mammography.

\section{Materials And Methods}

This is a prospective study in which 50 patients with clinically suspicious breast malignancy were taken over a period of 2 months. All the relevant clinical details were taken including age and mammographic findings were noted. Written informed consent was taken from all the patients. FNAC was done and routine Giemsa and pap stain was done. Additional two smears were fixed in cold acetone for p63 immunocytochemical staining. The mammography were reported using Breast Imaging Reporting Data Systems that includes BIRAD 1 ( negative),BIRAD 2 ( benign lesion), BIRAD 3 ( probably benign), BIRAD 4 (suspicious of malignancy) and 
BIRAD 5 (highly suggestive of malignancy). Cytodiagnosis was made by two different pathologists into different categories such as proliferative breast disease without atypia proliferative breast disease with atypia, atypical ductal cells and infiltrating ductal carcinoma (IDC). p63 staining was done using Ready to use antibody of Biogenex company. Only nuclear immunoreactivity was considered specific and quantified according to percentage of positive epithelial cell clusters and bare nuclei in the background. p63 grading was done as:- $0 \%, 1-25 \%, 26-50 \%, 51-75 \%$ and 76-100\% (1) .Diagnostic accuracy of cytological diagnosis and mammographical studies in atypical and malignant cases were compared using chi-square test of significance. Biopsies from subsequent operations on these cases were reviewed for correlation with the FNAC results.

\section{Results}

50 cases of clinically suspicious breast malignancy were taken over a period of 2 months. The clinical details, mammographic findings,FNAC diagnosis and histological diagnosis were studied. The age range of the patients was 31-70 years with maximum cases in 31-40 years age group (64\%) as shown in Table 1. Out of 50 clinically suspicious cases.27 cases (54\%) were cytologically diagnosed as ductal carcinoma followed by 16 cases $(32 \%)$ proliferative breast disease without atypia (Table /Figure 5). There was one case which was given as atypical ductal cells and was difficult to categorise on cytology.

Mammographic findings were compared with cytodiagnosisand p63 immuno-cyto staining as well (Figure 6).Out of 16 proliferative breast disease without atypia, 12 cases (75\%) were given BIRADS 3 on mammography. Out of 27 IDC cases, 18 cases( $66.67 \%$ ) were BIRADS 5 on mammography (Table 2). Cytological analysis of p63 stained slides showed a distinctive nuclear staining pattern. Out of 27 IDC cases, 26 $(96.29 \%)$ cases showed zero p63 positivity. 9 cases $(56.62 \%)$ out of 16 proliferative breast disease without atypia showed 76-100\% p63 positive cells (Table 3). The difference in the percentage of p63 positive epithelial cellclusters between benign and malignant lesions was statistically significant ( $p$-value $<0.005)$ (Table 4$)$.

\begin{tabular}{|l|c|l|}
\hline Age Range (years) & Number of Patients & Percentage \\
\hline $31-40$ & 32 & $64 \%$ \\
\hline $41-50$ & 12 & $24 \%$ \\
\hline$>50$ & 06 & $12 \%$ \\
\hline Total & 50 & \\
\hline
\end{tabular}

Table 1/Figure 1: Age wise distribution of patients

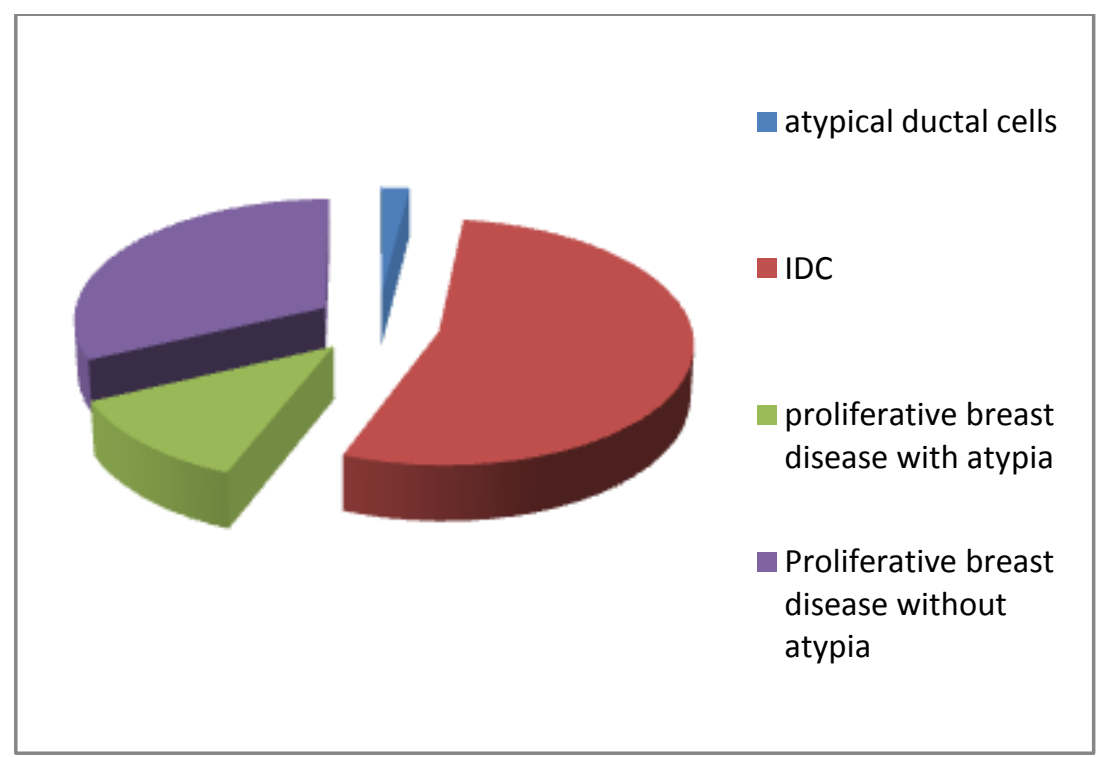

Table/Figure 5: Cyto-diagnosis of Breast Lesions 
Diagnostic Accuracy Of Mammography Versus Distribution Of P63 In Fine Needle Aspiration.....

\begin{tabular}{|l|l|c|c|}
\hline Sr no. & Cyto-diagnosis & Number of cases & $\begin{array}{c}\text { Mammography } \\
\text { (BIRADS) }\end{array}$ \\
\hline 1 & Proliferative breast disease without & 03 & II \\
& atypia $\quad(\mathrm{n}=16)$ & 12 & III \\
& & 01 & IV \\
\hline 2 & Proliferative breast disease with & 01 & III \\
& atypia $\quad(\mathrm{n}=06)$ & 04 & IV \\
& & 01 & V \\
\hline 3 & Atypical ductal cells (n=1) & 09 & IV \\
\hline 4 & Infiltrating ductal carcinoma (IDC) & 18 & V \\
\hline & Total & 50 & \\
\hline
\end{tabular}

Table 2/Figure 2: Correlation Of Mammography And Cyto-Diagnosis

\begin{tabular}{|c|c|c|c|c|c|}
\hline Cyto diagnosis & $0 \%$ & $1-25 \%$ & $26-50 \%$ & $51-75 \%$ & $76-100 \%$ \\
\hline $\begin{array}{l}\text { IDC } \\
(\mathrm{n}=27)\end{array}$ & $\begin{array}{c}26 \\
(96.29 \%)\end{array}$ & $\begin{array}{c}01 \\
(3.71 \%)\end{array}$ & 00 & 00 & 00 \\
\hline $\begin{array}{l}\text { Atypical ductal cells } \\
(\mathrm{n}=01)\end{array}$ & $\begin{array}{c}01 \\
(100 \%)\end{array}$ & 00 & 00 & 00 & 00 \\
\hline $\begin{array}{l}\text { Proliferative breast disease } \\
\text { with atypia } \\
\qquad(\mathrm{n}=06)\end{array}$ & 00 & $\begin{array}{c}02 \\
(33.33 \%)\end{array}$ & $\begin{array}{l}\mathbf{0 3} \\
(\mathbf{5 0} \%)\end{array}$ & $\begin{array}{c}01 \\
(16.67 \%)\end{array}$ & 00 \\
\hline $\begin{array}{l}\text { Proliferative breast disease } \\
\text { without atypia } \\
\qquad(\mathrm{n}=16)\end{array}$ & 00 & 00 & $\begin{array}{c}02 \\
(12.5 \%)\end{array}$ & $\begin{array}{c}05 \\
(31.25 \%)\end{array}$ & $\begin{array}{c}09 \\
(56.62 \%)\end{array}$ \\
\hline
\end{tabular}

Table 3/Figure 3: Correlation of cyto - diagnosis with p63 immunostaining

\begin{tabular}{|c|c|c|}
\hline Cyto - diagnosis & p63 percentage (zero) & Mammography (BIRADS 4/5) \\
\hline Benign & 00 & 06 \\
\hline IDC & 26 & 27 \\
\hline
\end{tabular}

Table 4/Figure 4: Correlation of p63 immunostain versus mammography $P$ value is 0.021 and is significant at $\mathrm{p}<0.05$.

\section{Discussion}

Breast cancer, a common malignant tumour is one of the leading cause of death amongst women (4). FNAC being simple, low cost, rapid method and gives accurate diagnosis in various benign and malignant lesions which makes FNAC an ideal initial diagnostic modality (6). Women with palpable breast lump on clinical examination are further investigated by imaging modalities such as mammography or ultrasound or even both. Choice of imaging technique partly depends on the age of patient. It is generally followed that women less than 35 years of age are investigated primarily by ultrasound and more than 35 years of age by mammography (7). With advent of time the constellation of clinical examination, radiological findings and cytopathological findings (referred as Triple test) is used for diagnosis of breast lesions as it has 100\% diagnostic accuracy (6).

This prospective study was undertaken in Department of cytopathology over a period of 2 months in which 50 clinically suspicious cases underwent FNAC along with additional smears fixed for immunocytology along with their mammographic findings. The age range of the patients was 31-70 years with maximum number of cases (64\%) in 31-40 years group followed by $24 \%$ in $41-50$ years age group. Our findings were corresponding to the findings of Tiwari M in a study of 91 breast lesions FNAC in which mean age was found to be 32 years (8). Another study done by Ahmed Omer et al in Sudan suggested that patients aged 30 years and older are more likely to be diagnosed malignant as they represented $96.78 \%$ of total malignant cases (9). Our study also endorsed the previous trend that there is strong association between breast cancer and older age.

Cytomorphological pattern was analysed in our study and IDC was most common lesion observed amongst suspicious cases accounting for $54 \%$ followed by proliferative breast lesion without atypia in 32\%, proliferative breast disease in $12 \%$ and one case as atypical ductal cells. All the clinically and mammographically benign lesions were excluded from doing FNAC. Our findings were in concordance with study by Nasreen $\mathrm{H} \mathrm{Hafez} \mathrm{in}$ which $81.6 \%$ cases were found to be malignant (1). 
Cytodiagnosis was also compared to mammographic findings. It was observed that 12 cases out of 16 proliferative breast disease without atypia were given BIRAD 3, 3 cases were BIRAD 2 and one case was given BIRAD 4.66.67\% cases of proliferative breast disease with atypia were BIRAD 4 and 16.67\% was given BIRAD $5.66 .67 \%$ IDC cases on cytology were given BIRAD 5 and $33.33 \%$ were BIRAD 4. Considering mammography and cytology together,it would appear that both techniques are equally accurate in predicting benign versus malignant diseases for lesions that are in the mammographical categories of BIRADS 1-3. For lesions in category BIRADS 4, cytology was a better predictor of which lesions were benign or malignant.

There are certain breast lesions that lie in "the gray zone" in which an unequivocal diagnosis of benignity or malignancy cannot be reached alone on cytology as these lesions show many overlapping features (10). The diagnosis of benign or malignant is based on identification of myoepithelial cells in papanicolaou stain in cytology. It becomes difficult at times to identify myoepithelial cells due to some obscuring factors. Therefore arises the need for immunochemical markers which act as an adjunct to FNAC (10). Keeping the biphenotypic property in mind (epithelial and smooth muscle like) many antibodies like smooth muscle actin, calponin, h-caldesmone are known. The drawback with all these is they can cross react with breast stromal cells,myofibroblast and neoplastic cells as well (11).

p63, recently characterised myoepithelial marker is considered to be gold standard myoepithelial marker in breast lesions by some authors. It is more specific as p63 being a nuclear stain aids in easy interpretation by overcoming the cytoplasmic fragility of myoepithelial cells in FNAC (12).p63, a p53 homologue has its gene located on $3 q 27-28$. It encodes six different proteins regulating epithelial proliferation and differentiation (11). Cytological analysis of p63 slides showed a distinctive nuclear staining pattern in myoepithelial cells. Out of 27 IDC cases,26 cases $(96.29 \%)$ showed no nuclear p63 staining in the epithelial cell clusters. There was one case reported as IDC on cytology which showed $1-25 \%$ p63 positive nuclear staining. This case when followed histologically was ductal carcinoma in situ. Our findings were corresponding to findings by other authors who stated 2 out of 17 malignant cases, one invasive and one DCIS, showed $50 \%$ or more p63 positive cell clusters. They also notified that in invasive cases, cytologically ductal tumour cells were p63 positive rather than myoepithelial cells (13). Mattia et al and Kaufmann et al reported 5-15\% of p63 positive neoplastic cells in $4.6 \%$ and $11 \%$ of breast carcinoma respectively $(14,15)$. The possible explanation could be partial myoepithelial/ basal differentiation of carcinoma breast.(16).

Lesions such as proliferative breast disease with/without atypia showed variable p63 positivity. None of the lesion from both the categories showed zero $\%$ p63 positivity. This result of our study is in line with the literature. There was one case reported as atypical ductal cells on cytology which showed zero percent p63 staining. Mammographically this case was BIRADS 5 and histologically it came out to be IDC. This case highlights the drawback of aspiration cytology.

When p63 findings were correlated with mammographic findings the accuracy of p63 immunocytostaining was significantly higher than mammography (p value $<0.05$ ). Mammographically 6 cases given as BIRAD4/5 were histologically benign, whereas none of the benign case showed zero p63 staining highlighting the improved accuracy of p63 stain in aiding the diagnosis. All the 27 IDC cases were given BIRADS 4/5 on mammography and as already discussed 26 cases showed zero p63 positivity.

\section{Conclusion}

It is important for both pathologists and clinicians to understand not only the benefits but also the limitations of cytological diagnosis from FNA specimens. Immediate evaluation of specimen adequacy is useful to eliminate equivocal diagnosis caused by technical factors. Combining cytology with p63 staining improved the diagnostic accuracy but the quantification of p63 positive myoepithelial cells needs to be standardised. Our study also did not attempt to draw any conclusion as to whether one diagnostic modality could replace the other.

\section{Acknowledgements}

None 


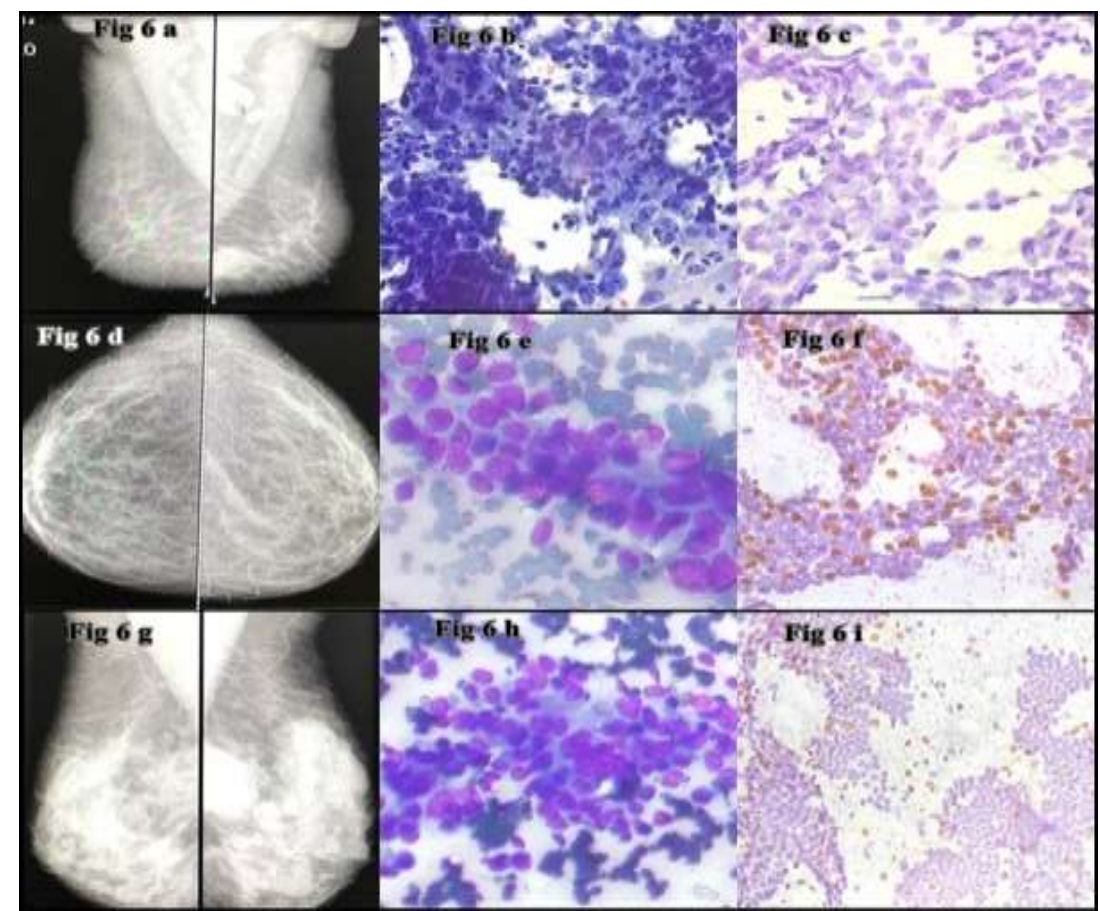

Table /Figure 6:

6a: BIRADS 5 Mammography, 6b: IDC, Pap stain 40X, 6c: p63 immunostain 40X - zero \% positivity. 6d: BIRADS 2 Mammography, 6e: Proliferative breast disease without atypia, Giemsa stain 40X, 6f: p63 immunostain 40X-76-100\% positivity.

6g: BIRADS 4 Mammography, 6h: Proliferative breast disease with atypia, Giemsa stain 40X, 6i: p63 immunostain 40X-25-50\% positivity.

\section{References}

[1]. Nasreen H Hafez, Neveen S Tahoun. The role of p63 immunocytochemistry for myoepithelial cells in the diagnosis of atypical and suspicious cases in Breast Fine needle aspiration cytology. Journal of the Egyptian Nat Cancer Inst,22(2), 2010,123-134.

[2]. B Chaiwan, N Sukhmwang, S Lekawanjit, K Sukapan, S Rangdaeng, M Muttarak, P S Thorner. Atypical and suspicious categories in fine needle aspiration cytology of the breast: histological and mammographical correlation and clinical significance. Singapore Med J 46(12),2006,706-709.

[3]. Taeko Nagao, Yoshimi Bando, Mitsunori Sasa, Tadaoki Morimoto, Nobuya Sano, Toshiyanki Hirose, Akira Tangoku. False positives in Fine needle aspiration cytology of breast disease can be reduced with p63 immunostaining- A preliminary report. Anticancer Research 26,2016, 4373-4378.

[4]. Rahman MZ, Sikdar AM, Nabi SR. Diagnosis of breast lump by fine needle aspiration cytology and mammography. Mymensingh Med J20(4), 2011,658-64.

[5]. Arisio R, Cuccorese C, Accinelli G, et al. Role of fine-needle aspiration biopsy in breast lesions: Analysis of a series of 4110 cases. Diagn Cytopathol.18, 1998, 462-7.

[6]. Florentine BD, Staymate B, Rabadi M. The reliability of fine-needle aspiration biopsy as the initial diagnostic procedure for palpable masses. Cancer 107, 2006,406-16.

[7]. Dixon J.M., Mansel R.E. Symptoms, assessment, and guidelines for referral. In: Dixon J.M., ed. (ABC of breast diseases, 2nd ed. London: BMJ 2000:3-7).

[8]. Tiwari M. role of Fine needle aspiration cytology in diagnosis of breast lumps. Kathmandu University Medical Journal 5(2),2007,215-17.

[9]. Ahmed Omer Almobarak, Taiseer Mohamed Elhassan, Mohamed Hasan Elhoweris, Heitham Mohammed Awadalla, Wadie Mohamed Yasin Elmadhoun, Mohamed Hassan Ahmed. Cytomorphologic patterns of Breast lesions in Sudanese patients: Lessons learned from fine needle aspiration cytology. Asian Pac J Cancer Prev 15(8),2014,3411-13.

[10]. Silverman JF, Elsheikh TM, Singh HK. The Role of Fine Needle Aspiration Cytology of the Breast in the Core Biopsy Era. Pathology Case Review.12(1), 2007, 44-8.

[11]. Tse GMK, Tan PH, Lui PCW, Gilks CB, Poom CSP, Ma TKF, Lam WWM. The role of immunohistochemistry for smooth muscle actin, p63, CD 10, and cytokeratin 14 in the differential diagnosis of papillary lesions of the breast. J Clin Pathol. 60,2007, 315-20.

[12]. Batistatou A, Stefanou D, Arkoumani E, Agnabtis NJ. The usefulness of p63 as a marker of breast myoepithelialcells. In Vivo.17(6), 2003, 573-6.

[13]. Harton AM, Wang HH, Schnitt SJ, Jacobs TW. P63 immunocytochemistry improves accuracy of diagnosis with fine needle aspiration of the breast. Am J Clin Pathol.128(1), 2007, 80-5.

[14]. Mattia B, Lorenza P, Giulia CM, Ettore M, Aroldo R, Giuseppe V, Claudio D. P63, a p53 Homologue, Is a selective nuclear marker of myoepithelial cells of human breast. American J Surg Pathol.25, 2001,1054-60. 
Diagnostic Accuracy Of Mammography Versus Distribution Of P63 In Fine Needle Aspiration.....

[15]. Kaufmann O, Fietze E, Mengs J, Dietel M. Value of p63 and cytokeratin 5/6 as immunohistochemical markers for the differential diagnosis of poorly differentiated and undifferentiated carcinomas. Am J Clin Pathol. 116,2001, 823-30.

[16]. Reis-Filho JS, Milanezi F, Amendoeira I, Albergaria A, Schmitt FC. P63 staining of myoepithelial cells in breast fine needle aspirates: A study of its role in differentiating in situ from invasive ductal carcinomas of the breast. J Clin Pathol.55(12), 2002, 9369. 